\title{
Predicting radiation pneumonitis after radiotherapy or chemoradiotherapy for oesophageal cancer: a single- center retrospective analysis
}

\section{Feng Du}

Shandong University Cheeloo College of Medicine https://orcid.org/0000-0003-4708-5075

\section{Wei Wang}

Shandong Cancer Hospital: Shandong Cancer Hospital and Institute

\section{Yankang Li}

Shandong University School of Medicine: Shandong University Cheeloo College of Medicine

\section{Yingjie Zhang}

Shandong Cancer Hospital and Institute

Jianbin Li ( $\sim$ lijianbin@msn.com )

Shandong Cancer Hospital Affiliated to Shandong Universitiy, Shandong Cancer Hospital and Institute, Shangdong

First Medical University and Shangdong Academy of Medical Sciences https://orcid.org/0000-0002-4053-1792

\section{Research}

Keywords: oesophageal cancer (EC), radiation pneumoniti (RP), radiotherapy (RT), chemotherapy

Posted Date: April 9th, 2021

DOI: https://doi.org/10.21203/rs.3.rs-388366/v1

License: (c) (i) This work is licensed under a Creative Commons Attribution 4.0 International License. Read Full License 


\section{Abstract}

Background: Multimodality therapy for oesophageal cancer (EC) can cause a variety of treatment-related sequelae, especially pulmonary toxicities. The accurate prediction of radiation pneumonitis $\triangle R P \bigotimes$ is essential to facilitate individualized radiation dosing that leads to maximized therapeutic gain. In this study, we performed a retrospective analysis to determine important factors that predict RP after radiotherapy (RT) for thoracic segment EC.

Methods: Two hundred and forty-seven patients with locally advanced EC who received RT or chemoradiotherapy $\triangle C R T \unrhd$ were enrolled. The factors associated with RP in different grades were analyzed by univariate and multivariate analyses, such as basic pulmonary disease, smoking index(SI), three mainstream RT techniques, and dose-volume histogram(DVH).

Results: The median RT dose was 60Gy, and the median follow-up time was 10 months. There were 118 cases of RP in 247 cases of EC patients who underwent RT or CRT. Among them, there were 54 cases of symptomatic pneumonitis ( $\geq 2$ grade). The overall rate of symptomatic pneumonitis was $21.9 \%$. In terms of RT techniques, there was no significant difference in the incidence of RP among three dimensional conformal radiotherapy (3D-CRT), intensity modulated radiotherapy (IMRT), and tomography (TOMO) $(\mathrm{P}>0.05) . \mathrm{V}_{5}-\mathrm{V}_{40}$ and MLD were associated with all grades of $R P \otimes P<0.05)$. Target volume, lung volume and their ratio were correlated with the incidence of $R P \otimes P<0.05)$. Among clinical factors, the highest risk of $\mathrm{RP} \otimes \geq 3$ grade $\$ was in patients $>400$ of SI. Chronic obstructive pulmonary disease (COPD) is also related to the occurrence of $\mathrm{RP}$ ( $\geq 1$ grade). In addition, $\mathrm{V}_{5}$ and $\mathrm{V}_{40}$ were an independent risk factor for RP grade $\geq 1$ (AUC 55.74\%, 4.13\%). MLD was an independent risk factor for RP grade $\geq 2$ (AUC 11.91Gy). $V_{5}$ was an independent risk factor for RP grade $\geq 3$ (AUC 57.60\%).

Conclusions: There was no significant difference in the incidence of RP regardless of radiation therapy technique (3DCRT, IMRT , and TOMO) and treatment-related factors. SI and COPD are closely related to the occurrence of the corresponding grades of RP. Several lung dosimetric parameters $\left(V_{5}, V_{40}, M L D\right)$ may be the most effective predictive factor of RP.

\section{Introduction}

Oesophageal cancer (EC) ranked as the seventh most frequently diagnosed cancer and the sixth leading cause of cancer-related death in 2018 worldwide ${ }^{[1]}$. Currently, radiotherapy (RT) is still the main treatment for locally advanced EC. It is an essential part of neoadjuvant chemoradiotherapy for operable locally advanced EC or radical chemoradiotherapy for inoperable $E C^{[2]}$. Radiation pneumonitis (RP) is one of the significant complications of thoracic RT. This has become the primary reason for limiting the dose increase of $\mathrm{RT}^{[3]}$. Although the clinical features are similar and the radiation dose is the same, the risk of RP in patients is different ${ }^{[4]}$. However, dosimetric parameters and clinical features are still commonly used in RP prediction ${ }^{[5]}$. The improvement of RT technology not only improves the local control rate of the tumour but also changes the lung dose distribution ${ }^{[6-7]}$. At present, the mainstream RT technology for EC is image-guided precise therapy, including three dimensional conformal radiotherapy (3D-CRT), intensity modulated radiotherapy (IMRT), and tomography (TOMO). The reduction in lung radiation dose will lay the foundation for the reduction in RP. Therefore, it is important to explore the correlation between different radiation techniques and RP. In this study, retrospective analysis was used to summarize the clinical data of 247 EC patients who underwent precise RT to determine and predict the factors related to RP and provide a basis for optimized RT and treatment for thoracic segment EC patients.

\section{Materials And Methods}




\section{Patients}

This is a retrospective single institutional study. The study was conducted in accordance with the ethical standards of Declaration of Helsinki and was approved by the institutional research ethics board of Shandong Cancer Hospital and Institute (SDTHEC201703014). A waiver of patient consent was granted because the research involved no more than minimal risk to the participants and the waiver will not adversely affect the rights and welfare of the participants. The study identified 247 thoracic segment EC patients who were diagnosed by endoscopic pathology from June 2014 to June 2019. The inclusion criteria were as follows: (1) no previous history of thoracic RT, (2) Karnofsky performance score (KPS) $\geq 70$, (3) all the patients receive radical RT, (4) availability of dosimetric parameters and clinical features, and (5) complete follow-up data and continuous follow-up time $\geq$ ten months. The exclusion criteria were as follows: (1) history of surgical resection of EC; (2) general pulmonary infection unrelated to RT; (3) treatment break of more than seven days during RT; and (4) incomplete radiotherapy follow-up data. There were 195 males (78.9\%) and 52 females $(21.1 \%)$ with an average age of 64 years (range $41-87$ years). Pathological features: 237 cases $(96.0 \%)$ of squamous cell carcinoma, 6 cases $(2.4 \%)$ of adenocarcinoma, and 4 cases $(1.6 \%)$ of small cell carcinoma. Clinical stage (II: 34 cases, 13.8\%; III: 130 cases, 52.6\%, 邓: 83 cases, 33.6\%). Patients underwent 3DCRT ( $=21 ; 8.5 \%)$, IMRT (n $=161 ; 65.2 \%)$, and TOMO $(n=65 ; 26.3 \%)$.

\section{Basic requirements of RT}

The target design is based on simulated localization contrast-enhanced CT for diagnostic examination results, such as endoscopic ultrasonography and positron emission tomography (PET). The target area includes gross tumour volume (GTV), CT imaging visible oesophageal tumour and positive lymph nodes. The clinical target volume (CTV) is the upper and lower outward expansion of the oesophageal tumour and related lymphatic drainage area. The planned target volume (PTV) has an outward expansion of the CTV of 6-8 $\mathrm{mm}$. In addition, there is no standard for target delineation for the volume range of CTV/PTV, since we consider the patient's physical condition (KPS, age), N stage (how many lymph nodes metastases), and other factors. We have given two different irradiation methods, namely, elective node irradiation (ENI) and involved-field irradiation (IFI), resulting in different CTV/PTV sizes.

IMRT adopts a fixed field static intensity modulation technique, and 5-7 fields of coplanar irradiation fields are uniformly divided according to the specific situation of the case. The required target parameters are then set, and the dose distribution is obtained by inverse calculation of the treatment planning system. The dose distribution was then graded (stratified), and each field was decomposed into a series of subfields. IMRT does not include simplified intensity-modulated radiotherapy (sIMRT) or volumetric intensity-modulated arc therapy (VMAT). TOMO adopts the spiral TOMO intensity modulation technique, and each spiral contains 51 beams. 3DCRT and IMRT plans were generated in the Eclipse system (Varian Medical Systems, Palo Alto, CA, Version 13.5.35), and TOMO plans were generated in the TomoTherapy® Planning Station (Accuray, Sunnyvale, CA, Hi-Art, Version 5.1.3). 3DCRT and IMRT were administered by a Varian Linac Accelerator with 6-MVX ray and 95\% PTV and radiation doses of 50-66 Gy (median dose of $60 \mathrm{~Gy}$ ) and 1.8-2.0 Gy/fraction, five times a week were prescribed. Normal tissue constraints were prioritized in the following order for treatment planning purposes: maximum spinal cord dose of $45 \mathrm{~Gy}$, relative volume of total lung treated with $\geq 5 \mathrm{~Gy}\left(\mathrm{~V}_{5}\right) \leq 60 \%$, relative volume of total lung treated with $\geq 20 \mathrm{~Gy}\left(\mathrm{~V}_{20}\right) \leq 28 \%$, mean lung dose $(M L D) \leq 20 \mathrm{~Gy}$, relative volume of the heart treated with $\geq 30 \mathrm{~Gy}\left(\mathrm{~V}_{30}\right) \leq 40 \%$, and relative volume of the heart treated with $\geq 40$ Gy $\left(\mathrm{V}_{40}\right) \leq 30 \%$.

\section{Chemotherapy regimens}

Most patients separately received induction, concurrent, or sequential chemotherapy based on an individualized treatment strategy. The chemotherapy regimens included cisplatin with fluorouracil (PF regimen) and cisplatin with 
paclitaxel (TP regimen). In all patients, 42 patients (17.0\%) did not receive chemotherapy, 97 patients (39.3\%) received induction chemotherapy, 21 patients (8.5\%) received induction and concurrent chemoradiotherapy, 62 patients (25.1\%) received concurrent chemoradiotherapy, and 25 patients $(10.1 \%)$ received consolidation chemotherapy after RT. The median number of chemotherapy cycles was 4 (range, $2 ₫ 6$ cycles). The doses and adjustments of chemotherapy regimens followed the guidelines of the National Comprehensive Cancer Network (NCCN) for EC.

\section{Staging criteria and segments}

(1)EC staging criteria: The American Joint Commission on Cancer (AJCC) TNM Staging System (8th Edition); (2) segments: The distance between the upper incisor and the epicenter of the tumour is used to indicate the specific position. Upper thoracic oesophagus: from the thoracic entrance to the lower edge of the azygos vein arch, endoscopic examination 20-25 cm away from the incisor. Middle thoracic oesophagus: from the lower edge of the zygos vein arch to the level of the inferior pulmonary vein, endoscopic examination is $25-30 \mathrm{~cm}$ away from the incisor. Lower thoracic oesophagus: from the level of the lower pulmonary vein down to the gastroesophageal junction, endoscopy $30-35 \mathrm{~cm}$ away from the incisor.

\section{Observation index}

The observation index includes sex, age, tumour stage, SI, COPD, chemotherapy regimen, and dosimetric parameters. The dose parameters included total radiation dose, lung volume, target volume, MLD, and the relative volume of the total lung treated with $\geq 5 \sim 40 \mathrm{~Gy}\left(\mathrm{~V}_{5} \sim \mathrm{V}_{40}\right)$.

\section{Follow-up and Evaluation of RP}

The follow-up items included chest CT, physical examination and the clinical symptoms. Patients were evaluated weekly during RT, followed up at one month after completion of the initial treatment and then were followed up every 2-3 months, at least until half a year after the end of RT. The diagnosis and classification of RP were confirmed by two senior radiologists (professional years $\geq 5$ years) and one radiologist. In addition, while some patients may have varying degrees of RP at different points in time, the RP grade we provide is when patients have the most severe RP during follow-up. The RP was graded by the Common Termination Criteria for Adverse Events, version 4.0.3 (CTCAE 4.0.3).The diagnostic criteria for RP were as follows: Grade 1: Asymptomatic; clinical or diagnostic observations only; intervention not indicated; Grade 2: Symptomatic; medical intervention indicated; limiting instrumental activities of daily living (ADL); Grade 3: Severe symptoms; limiting self-care ADL; oxygen indicated; Grade 4: Lifethreatening respiratory compromise; urgent intervention indicated; and Grade 5: Death.

\section{Statistical treatment}

All data were analysed using the statistical package for the Social Science program (SPSS for Windows, version 17.0, IBM, Armonk, NY, USA). The quantitative data were compared by ANOVA, and the count data were compared by $\chi^{2}$ tests or Fisher's exact probability test. Univariate analysis was conducted for all the influencing factors involved in this study. Multivariate analysis was performed for those with $\mathrm{P}<0.05$ after univariate analysis. The results of the multivariate analysis were all independent risk factors. Logistic regression analysis was used to analyse independent risk factors. The optimal cut-off values of parameters were calculated using receiver operating characteristic (ROC) curves. A value of two-sided $\mathrm{P}<0.05$ was considered statistically significant.

\section{Results}

Patient characteristics and incidence of RP in different grades 
The characteristics of the 247 patients included in this study are listed in Table 1. The follow-up time of all patients was more than six months; the median follow-up time was 11 months. Among them, 64 cases were grade 1 RP (25.9\%), 37 cases were grade 2 RP (14.9\%), 12 cases were grade 3 RP (4.9\%), 5 cases were grade 4 RP (0.2\%), and no cases were grade $5 \mathrm{RP}(0.0 \%)$. Fifty-four patients with RP grade $\geq 2$ were treated with oxygen therapy or \pm antibiotics or glucocorticoid (dexamethasone or methylprednisolone). Among them, 50 patients were improved or cured, and two patients were complicated with pulmonary insufficiency and severe pulmonary fibrosis, requiring long-term oxygen therapy. Three patients (one case with grade 2 and two cases grade $\geq 3$ ) died of respiratory failure due to severe infection and respiratory distress syndrome. There was no significant correlation in the risk of $\operatorname{RP}$ ( $g r a d e \geq 1, \geq 2, \geq 3$ ) between patient factors (sex, age, tumour stage, tumour location, diabetes, hypertension) and treatment factors (chemotherapy or lack thereof, chemotherapy mode or regimens, delivered RT dose, induction chemotherapy cycle). More importantly, there was no significant difference in the incidence of RP between 3DCRT, IMRT and TOMO (RP grade $\geq 1$ ( $47.6 \%$ vs $50.3 \%$ vs $41.5 \%$ ), grade $\geq 2$ (33.3\% vs $22.4 \%$ vs $16.9 \%)$, and grade $\geq 3(9.5 \%$ vs $7.5 \%$ vs $4.6 \%)$ ). Although there was no significant difference in the incidence of RP $\geq$ grade 1 and $\geq$ grade 2 between nonsmokers and smokers, the incidence of RP $\geq$ grade 3 in smokers with smoking index $>400$ was significantly higher $(P=0.001)$. In addition, COPD was significantly correlated with $R P \geq$ grade $1(P=0.003)$, as shown in Table 1 . Multivariate analysis showed that smoking index $>400$ was an independent risk factor for $R P \geq$ grade 3 (Wald $=5.964, P<0.05$ ), and COPD was an independent risk factor for $\mathrm{RP} \geq$ grade 1 (Wald $=6.110, \mathrm{P}<0.05$ ), as shown in Table 3. 
Table 1

Clinical characteristics and treatment model of RP patients of different grades

\begin{tabular}{|c|c|c|c|c|c|c|c|c|c|c|}
\hline Characteristics & $\mathbf{n}$ & $\begin{array}{l}\geq 1 \text { grade } \\
{[n,(\%)]}\end{array}$ & $\chi_{\text {value }}^{2}$ & $\begin{array}{l}P \text { - } \\
\text { value }\end{array}$ & $\begin{array}{l}\geq 2 \\
\text { grade } \\
{[n,(\%)]}\end{array}$ & $\begin{array}{l}\chi^{2} \\
\text { value }\end{array}$ & $\begin{array}{l}\mathrm{P}- \\
\text { value }\end{array}$ & $\begin{array}{l}\geq 3 \\
\text { grade } \\
\text { [n, (\%)] }\end{array}$ & $\begin{array}{l}\chi^{2} \\
\text { value }\end{array}$ & $\begin{array}{l}\text { P- } \\
\text { value }\end{array}$ \\
\hline \multicolumn{11}{|l|}{ Sex } \\
\hline Male & 195 & $91(46.7)$ & 0.455 & 0.534 & $37(19.0)$ & 4.522 & 0.039 & $12(6.2)$ & 0.764 & 0.382 \\
\hline Female & 52 & $27(51.9)$ & & & 17(32.7) & & & $5(9.6)$ & & \\
\hline \multicolumn{11}{|l|}{ Age (years) } \\
\hline$<60$ & 78 & $41(52.6)$ & 1.049 & 0.339 & $19(24.4)$ & 0.416 & 0.513 & $10(12.8)$ & 6.272 & 0.027 \\
\hline$\geq 60$ & 169 & $77(45.6)$ & & & $35(20.7)$ & & & $7(4.1)$ & & \\
\hline \multicolumn{11}{|l|}{ Smoking index } \\
\hline 0 & 137 & $68(49.6)$ & 2.676 & 0.262 & $29(21.1)$ & 5.976 & 0.050 & $6(4.4)$ & 13.295 & 0.001 \\
\hline $100 \rrbracket 400$ & 39 & 14(35.9) & & & $4(10.3)$ & & & $0(0)$ & & \\
\hline$>400$ & 71 & $36(50.7)$ & & & $21(29.6)$ & & & $11(15.5)$ & & \\
\hline \multicolumn{11}{|l|}{ Tumour stage } \\
\hline ॥ & 34 & $14(41.1)$ & 1.175 & 0.556 & $9(26.5)$ & 1.184 & 0.553 & $1(2.9)$ & 2.665 & 0.264 \\
\hline III & 130 & $61(46.9)$ & & & $25(19.2)$ & & & $12(9.2)$ & & \\
\hline IV & 83 & $43(51.8)$ & & & $20(24.1)$ & & & $4(4.8)$ & & \\
\hline \multicolumn{11}{|l|}{$\begin{array}{l}\text { Tumour } \\
\text { location }\end{array}$} \\
\hline Upper & 44 & $20(45.5)$ & 0.709 & 0.702 & $9(20.5)$ & 4.787 & 0.091 & $3(6.8)$ & 1.051 & 0.591 \\
\hline Middle & 152 & $71(46.7)$ & & & $39(25.7)$ & & & $12(7.9)$ & & \\
\hline Lower & 51 & $27(52.9)$ & & & $6(11.7)$ & & & $2(3.9)$ & & \\
\hline \multicolumn{11}{|l|}{ COPD } \\
\hline No & 212 & $93(43.9)$ & 9.146 & 0.003 & 42(19.8) & 3.684 & 0.075 & $14(6.6)$ & 0.181 & 0.671 \\
\hline Yes & 35 & $25(71.4)$ & & & $12(34.3)$ & & & $3(8.6)$ & & \\
\hline \multicolumn{11}{|l|}{ Diabetes } \\
\hline No & 217 & $103(47.5)$ & 0.068 & 0.847 & $47(21.7)$ & 0.043 & 0.816 & $15(6.9)$ & 0.002 & 0.960 \\
\hline Yes & 30 & $15(50.0)$ & & & $7(23.3)$ & & & $2(6.6)$ & & \\
\hline Hypertension & & & & & & & & & & \\
\hline
\end{tabular}

3DCRT = three-dimensional conformal radiotherapy; IMRT = intensity-modulated radiotherapy;TOMO = helical tomotherapy;RTT=radiotherapy techniques; $\mathrm{ICC}$ = induction chemotherapy cycle;PF = cisplatin with fluorouracil; TP = cisplatin with paclitaxel 


\begin{tabular}{|c|c|c|c|c|c|c|c|c|c|c|}
\hline Characteristics & $\mathrm{n}$ & $\begin{array}{l}\geq 1 \text { grade } \\
{[n,(\%)]}\end{array}$ & $\begin{array}{l}\chi^{2} \\
\text { value }\end{array}$ & $\begin{array}{l}\text { P- } \\
\text { value }\end{array}$ & $\begin{array}{l}\geq 2 \\
\text { grade } \\
{[n,(\%)]}\end{array}$ & $\chi_{\text {value }}^{2}$ & $\begin{array}{l}\text { P- } \\
\text { value }\end{array}$ & $\begin{array}{l}\geq 3 \\
\text { grade } \\
{[n,(\%)]}\end{array}$ & $\chi_{\text {value }}^{2}$ & $\begin{array}{l}\text { P- } \\
\text { value }\end{array}$ \\
\hline No & 202 & $99(49.0)$ & 0.680 & 0.510 & $45(22.3)$ & 0.112 & 0.843 & $14(6.9)$ & 0.004 & 0.950 \\
\hline Yes & 45 & $19(42.2)$ & & & $9(20.0)$ & & & $3(6.6)$ & & \\
\hline \multicolumn{11}{|l|}{ RTT } \\
\hline 3DCRT & 21 & $10(47.6)$ & 1.434 & 0.488 & $7(33.3)$ & 2.469 & 0.291 & $2(9.5)$ & 0.871 & 0.647 \\
\hline IMRT & 161 & $81(50.3)$ & & & $36(22.3)$ & & & $12(7.5)$ & & \\
\hline TOMO & 65 & $27(41.5)$ & & & $11(16.9)$ & & & $3(4.6)$ & & \\
\hline \multicolumn{11}{|l|}{$\begin{array}{l}\text { Delivered dose } \\
\text { (Gy) }\end{array}$} \\
\hline$<60$ & 74 & $30(40.5)$ & 2.215 & 0.165 & 14(18.9) & 0.536 & 0.506 & $2(2.7)$ & 2.880 & 0.105 \\
\hline$\geq 60$ & 173 & $88(50.9)$ & & & $40(23.1)$ & & & $15(8.7)$ & & \\
\hline \multicolumn{11}{|l|}{$\begin{array}{l}\text { Chemotherapy } \\
\text { mode }\end{array}$} \\
\hline No & 42 & $20(47.6)$ & 3.672 & 0.452 & $12(28.6)$ & 4.928 & 0.295 & $2(4.7)$ & 4.793 & 0.309 \\
\hline Induce & 97 & $51(52.6)$ & & & $25(25.8)$ & & & $7(7.2)$ & & \\
\hline Concurrent & 62 & $30(48.4)$ & & & 11(17.7) & & & $6(9.7)$ & & \\
\hline $\begin{array}{l}\text { Induce + } \\
\text { Concurrent }\end{array}$ & 21 & $9(42.9)$ & & & $3(14.3)$ & & & $2(9.5)$ & & \\
\hline Adjuvant & 25 & $8(32.0)$ & & & $3(12.0)$ & & & $0(0.0)$ & & \\
\hline \multicolumn{11}{|l|}{$\begin{array}{l}\text { Chemotherapy } \\
\text { regimen }\end{array}$} \\
\hline No & 42 & $20(47.6)$ & 2.054 & 0.358 & $12(28.6)$ & 3.388 & 0.184 & $2(4.8)$ & 4.437 & 0.109 \\
\hline TP & 132 & $68(51.5)$ & & & $31(23.5)$ & & & $13(9.8)$ & & \\
\hline PF & 73 & $30(41.1)$ & & & 11(15.1) & & & $2(2.7)$ & & \\
\hline \multicolumn{11}{|l|}{ ICC(NO.) } \\
\hline $0 \llbracket 3$ & 203 & 93(45.8) & 1.755 & 0.244 & $40(19.7)$ & 3.106 & 0.106 & $14(6.9)$ & 0.000 & 0.985 \\
\hline$\geq 3$ & 44 & $25(56.8)$ & & & 14(31.8) & & & $3(6.8)$ & & \\
\hline \multicolumn{11}{|c|}{ 3DCRT = three-dimensional conformal radiotherapy; IMRT = intensity-modulated radiotherapy; $T O M O=$ helical } \\
\hline \multicolumn{11}{|c|}{ tomotherapy;RTT=radiotherapy techniques;ICC = induction chemotherapy cycle;PF = cisplatin with fluorouracil; } \\
\hline TP = cisplatin wit & pacli & cel & & & & & & & & \\
\hline
\end{tabular}


Table 3

Multivariate analysis results of risk factors for patients with different grades of RP

\begin{tabular}{|c|c|c|c|c|c|c|c|c|}
\hline \multirow[t]{2}{*}{ Grade } & \multirow[t]{2}{*}{ Risk factors } & \multirow[t]{2}{*}{$B$} & \multirow[t]{2}{*}{ Standard error } & \multirow[t]{2}{*}{ Wald } & \multirow[t]{2}{*}{$P$-value } & \multirow[t]{2}{*}{$E X P(B)$} & \multicolumn{2}{|c|}{$\operatorname{EXP}(B)-95 \% \mathrm{Cl}$} \\
\hline & & & & & & & lower limit & upper limit \\
\hline \multirow[t]{3}{*}{$\mathrm{RP} \geq 1$} & COPD & 1.102 & 0.446 & 6.110 & 0.013 & 1.332 & 1.017 & 5.739 \\
\hline & $\mathrm{V}_{5}$ & 0.054 & 0.029 & 3.427 & 0.041 & 1.055 & 1.004 & 1.095 \\
\hline & $\mathrm{V}_{40}$ & 0.319 & 0.155 & 4.250 & 0.039 & 1.375 & 1.016 & 1.862 \\
\hline $\mathrm{RP} \geq 2$ & MLD(Gy) & 0.253 & 0.150 & 2.832 & 0.012 & 1.289 & 1.059 & 1.730 \\
\hline \multirow[t]{2}{*}{$\mathrm{RP} \geq 3$} & Smoking index $₫ 400$ & 1.443 & 0.591 & 5.964 & 0.015 & 4.233 & 1.330 & 13.479 \\
\hline & $\mathrm{V}_{5}$ & 1.541 & 0.628 & 6.013 & 0.014 & 1.241 & 1.063 & 1.734 \\
\hline
\end{tabular}

\section{Correlation between dose-volume parameters and the incidence of RP in different grades}

In the whole group, the mean (standard deviation) doses of $\mathrm{V}_{5}$ were $54.78 \pm 12.58(\%), \mathrm{V}_{10}$ was $36.98 \pm 9.91$ (\%), $\mathrm{V}_{15}$ was $28.04 \pm 7.29(\%), V_{20}$ was $20.44 \pm 5.62(\%), V_{30}$ was $10.75 \pm 4.58(\%), V_{40}$ was $4.77 \pm 3.03(\%)$, and the mean MLD dose was $11.21 \pm 3.06(\mathrm{~Gy})$. The mean lung volume was $3447.7 \pm 917.8\left(\mathrm{~cm}^{3}\right), \mathrm{GTV}$ was $144.42 \pm 116.38\left(\mathrm{~cm}^{3}\right)$, and PTV was $400.93 \pm 216.26\left(\mathrm{~cm}^{3}\right)$. Both $\mathrm{V}_{5} \sim \mathrm{V}_{40}$ and MLD were associated with the occurrence of RP $\geq$ grade 1 , $\geq$ grade 2 , and $\geq$ grade $3(P<0.05)$. Moreover, GTV, PTV, GTV/total lung volume, and PTV/total lung volume were all associated with $\mathrm{RP} \geq$ grade 1 and $\geq$ grade $2(\mathrm{P}<0.05)$, while total lung volume $\left(\mathrm{cm}^{3}\right)$ was not related to the occurrence of RP at any level $(P>0.05)$, as shown in Table 2. Multivariate analysis showed that $V_{5}$ and $V_{40}$ were independent risk factors for $R P \geq$ grade $1(P<0.05)$; $M L D$ and $V_{5}$ were independent risk factors for $R P \geq$ grade 2 and $\geq$ grade $3(P<0.05)$, respectively, as shown in Table 3. 
Table 2

Irradiation dose -volume parameters of patients with different grades of RP ( $X \pm S$ )

\begin{tabular}{|c|c|c|c|c|c|c|c|c|c|c|}
\hline \multirow[t]{2}{*}{ Parameter } & \multirow{2}{*}{$\begin{array}{l}\text { All } \\
(n= \\
247)\end{array}$} & \multicolumn{2}{|c|}{$\geq 1$ grade $R P$} & \multirow{2}{*}{$\begin{array}{l}P \\
\text { value }\end{array}$} & \multicolumn{2}{|c|}{$\geq 2$ grade $R P$} & \multirow{2}{*}{$\begin{array}{l}P \\
\text { value }\end{array}$} & \multicolumn{2}{|c|}{$\geq 3$ grade $R P$} & \multirow{2}{*}{$\begin{array}{l}P \\
\text { value }\end{array}$} \\
\hline & & $\begin{array}{l}\text { No }(n= \\
129)\end{array}$ & $\begin{array}{l}\text { Yes }(n \\
=118)\end{array}$ & & $\begin{array}{l}\text { No }(n= \\
193)\end{array}$ & $\begin{array}{l}\text { Yes }(n= \\
54)\end{array}$ & & $\begin{array}{l}\text { No }(n \\
=230)\end{array}$ & $\begin{array}{l}\text { Yes (n } \\
=17)\end{array}$ & \\
\hline $\mathrm{V}_{5}$ & $\begin{array}{l}54.78 \\
\pm 12.58\end{array}$ & $\begin{array}{l}50.39 \\
\pm 10.97\end{array}$ & $\begin{array}{l}59.57 \\
\pm 12.51\end{array}$ & 0.000 & $\begin{array}{l}52.40 \\
\pm 12.03\end{array}$ & $\begin{array}{l}63.25 \pm \\
10.79\end{array}$ & 0.000 & $\begin{array}{l}54.05 \\
\pm 12.43\end{array}$ & $\begin{array}{l}64.62 \\
\pm 10.49\end{array}$ & 0.001 \\
\hline $\mathrm{V}_{10}$ & $\begin{array}{l}36.98 \\
\pm 9.91\end{array}$ & $\begin{array}{l}33.87 \\
\pm 8.68\end{array}$ & $\begin{array}{l}40.38 \\
\pm 10.08\end{array}$ & 0.000 & $\begin{array}{l}35.27 \\
\pm 9.36\end{array}$ & $\begin{array}{l}43.12 \pm \\
9.45\end{array}$ & 0.000 & $\begin{array}{l}36.43 \\
\pm 9.85\end{array}$ & $\begin{array}{l}44.42 \\
\pm 7.59\end{array}$ & 0.001 \\
\hline $\mathrm{V}_{15}$ & $\begin{array}{l}28.04 \\
\pm 7.29\end{array}$ & $\begin{array}{l}25.68 \\
\pm 6.64\end{array}$ & $\begin{array}{l}30.62 \\
\pm 7.11\end{array}$ & 0.000 & $\begin{array}{l}26.77 \\
\pm 6.97\end{array}$ & $\begin{array}{l}32.59 \pm \\
6.60\end{array}$ & 0.000 & $\begin{array}{l}27.68 \\
\pm 7.31\end{array}$ & $\begin{array}{l}32.91 \\
\pm 4.97\end{array}$ & 0.004 \\
\hline $\mathrm{V}_{20}$ & $\begin{array}{l}20.44 \\
\pm 5.62\end{array}$ & $\begin{array}{l}18.54 \\
\pm 5.13\end{array}$ & $\begin{array}{l}22.52 \\
\pm 5.41\end{array}$ & 0.000 & $\begin{array}{l}19.36 \\
\pm 5.32\end{array}$ & $\begin{array}{l}24.30 \pm \\
4.98\end{array}$ & 0.000 & $\begin{array}{l}20.19 \\
\pm 5.61\end{array}$ & $\begin{array}{l}23.79 \\
\pm 4.74\end{array}$ & 0.011 \\
\hline$v_{30}$ & $\begin{array}{l}10.75 \\
\pm 4.58\end{array}$ & $\begin{array}{l}9.43 \pm \\
4.18\end{array}$ & $\begin{array}{l}12.20 \\
\pm 4.58\end{array}$ & 0.000 & $\begin{array}{l}9.89 \pm \\
4.32\end{array}$ & $\begin{array}{l}13.82 \pm \\
4.18\end{array}$ & 0.000 & $\begin{array}{l}10.53 \\
\pm 4.54\end{array}$ & $\begin{array}{l}13.69 \\
\pm 4.32\end{array}$ & 0.007 \\
\hline $\mathrm{V}_{40}$ & $\begin{array}{l}4.77 \pm \\
3.03\end{array}$ & $\begin{array}{l}3.79 \pm \\
2.26\end{array}$ & $\begin{array}{l}5.85 \pm \\
3.39\end{array}$ & 0.000 & $\begin{array}{l}4.13 \pm \\
2.50\end{array}$ & $\begin{array}{l}7.07 \pm \\
3.61\end{array}$ & 0.000 & $\begin{array}{l}4.57 \pm \\
2.88\end{array}$ & $\begin{array}{l}7.52 \pm \\
3.67\end{array}$ & 0.001 \\
\hline MLD(Gy) & $\begin{array}{l}11.21 \\
\pm 3.06\end{array}$ & $\begin{array}{l}10.18 \\
\pm 2.59\end{array}$ & $\begin{array}{l}12.33 \\
\pm 3.15\end{array}$ & 0.000 & $\begin{array}{l}10.54 \\
\pm 2.79\end{array}$ & $\begin{array}{l}13.59 \pm \\
2.80\end{array}$ & 0.000 & $\begin{array}{l}11.05 \\
\pm 3.03\end{array}$ & $\begin{array}{l}13.38 \\
\pm 2.65\end{array}$ & 0.002 \\
\hline $\operatorname{TLV}(\mathrm{cc})$ & $\begin{array}{l}3447.7 \\
\pm \\
917.82\end{array}$ & $\begin{array}{l}3512.4 \\
\pm \\
916.16\end{array}$ & $\begin{array}{l}3377.0 \\
\pm \\
918.20\end{array}$ & 0.248 & $\begin{array}{l}3486.0 \\
\pm \\
871.51\end{array}$ & $\begin{array}{l}3310.8 \\
\pm \\
1064.91\end{array}$ & 0.263 & $\begin{array}{l}3472.9 \\
\pm \\
923.05\end{array}$ & $\begin{array}{l}3107.4 \\
\pm \\
790.60\end{array}$ & 0.113 \\
\hline $\mathrm{GTV}(\mathrm{cc})$ & $\begin{array}{l}144.42 \\
\pm \\
116.38\end{array}$ & $\begin{array}{l}123.89 \\
\pm 85.71\end{array}$ & $\begin{array}{l}166.85 \\
\pm \\
139.53\end{array}$ & 0.041 & $\begin{array}{l}132.97 \\
\pm \\
100.86\end{array}$ & $\begin{array}{l}185.34 \\
\pm \\
154.37\end{array}$ & 0.029 & $\begin{array}{l}140.19 \\
\pm \\
107.29\end{array}$ & $\begin{array}{l}201.63 \\
\pm \\
199.38\end{array}$ & 0.421 \\
\hline $\mathrm{PTV}(\mathrm{cc})$ & $\begin{array}{l}400.93 \\
\pm \\
216.26\end{array}$ & $\begin{array}{l}357.09 \\
\pm \\
190.55\end{array}$ & $\begin{array}{l}448.86 \\
\pm \\
232.75\end{array}$ & 0.001 & $\begin{array}{l}375.50 \\
\pm \\
196.29\end{array}$ & $\begin{array}{l}491.81 \\
\pm \\
258.31\end{array}$ & 0.002 & $\begin{array}{l}393.20 \\
\pm \\
203.51\end{array}$ & $\begin{array}{l}505.55 \\
\pm \\
337.34\end{array}$ & 0.275 \\
\hline GTV/TLV & $\begin{array}{l}0.05 \pm \\
0.05\end{array}$ & $\begin{array}{l}0.04 \pm \\
0.03\end{array}$ & $\begin{array}{l}0.06 \pm \\
0.07\end{array}$ & 0.020 & $\begin{array}{l}0.04 \pm \\
0.05\end{array}$ & $\begin{array}{l}0.06 \pm \\
0.06\end{array}$ & 0.012 & $\begin{array}{l}0.05 \pm \\
0.05\end{array}$ & $\begin{array}{l}0.07 \pm \\
0.08\end{array}$ & 0.159 \\
\hline PTV/TLV & $\begin{array}{l}0.13 \pm \\
0.09\end{array}$ & $\begin{array}{l}0.11 \pm \\
0.08\end{array}$ & $\begin{array}{l}0.04 \pm \\
0.10\end{array}$ & 0.001 & $\begin{array}{l}0.12 \pm \\
0.08\end{array}$ & $\begin{array}{l}0.17 \pm \\
0.11\end{array}$ & 0.001 & $\begin{array}{l}0.13 \pm \\
0.08\end{array}$ & $\begin{array}{l}0.17 \pm \\
0.13\end{array}$ & 0.175 \\
\hline \multicolumn{11}{|c|}{$\begin{array}{l}\text { MLD = mean lung dose, V5, V10, V15, V20, V30, V40 = relative volume of total lung treated with } \geq 5,10,15,20,30 \text {, } \\
\text { and } 40 \mathrm{~Gy} ;\end{array}$} \\
\hline
\end{tabular}

\section{Predictive risk assessment models}

The receiver operating characteristic curves (ROCs) of $\mathrm{V}_{5}, \mathrm{~V}_{40}$ and the MLD were drawn to clarify the relationship between the dosimetric parameters and the risk of RP in the multivariate results, as shown in Fig. 1. The thresholds of $V_{5}$ and $V_{40}(R P \geq$ grade 1$)$ were $55.74 \%$ and $4.13 \%$, respectively. The AUCs of $V_{5}$ and $V_{40}(R P \geq$ grade 1$)$ were 0.714 (95\% Cl, 0.649-0.778, P<0.001) and 0.688 (95\% Cl, 0.622-0.754, $\mathrm{P}<0.001)$, respectively (Fig. 1A). The threshold of MLD (RP $\geq$ grade 2) was $11.91 \mathrm{~Gy}$. The AUC of MLD (RP $\geq$ grade 2$)$ was $0.773(95 \% \mathrm{Cl}, 0.703-0.844, \mathrm{P}<0.001)$ (Fig. 1B). The threshold of $\mathrm{V}_{5}(\mathrm{RP} \geq$ grade 3$)$ was $57.60 \%$. The $A U C$ of $\mathrm{V}_{5}$ (RP $\geq$ grade 3$)$ was $0.749(95 \% \mathrm{Cl}, 0.648-$ 0.850, $\mathrm{P}<0.001$ ) (Fig. 1C). 


\section{Discussion}

$\mathrm{RP}$ is an inflammation of normal lung tissue caused by radiation therapy ${ }^{[8]}$. If RP occurs, it will seriously affect the quality of life and survival prognosis. Therefore, it is imperative for EC patients undergoing RT to identify lung toxicity at the earliest possible time. The occurrence of RP is not the result of a single factor but is affected by multiple factors. Among these factors, the lung dose-volume parameter is still an essential indicator for RP prediction. In recent years, studies $^{[9,10]}$ have suggested that low-dose parameters such as $V_{5}$ and $V_{10}$ are the most critical factors related to RP. Jin et al. ${ }^{[11]}$ considered that the low-dose parameter $\mathrm{V}_{13}$ can also be used as a predictor of $\mathrm{RP}$, and $\mathrm{V}_{13}<40 \%$ can reduce the incidence of RP. Moreover, MLD is also one of the parameters concerned in thoracic RT. Several studies ${ }^{[12,}$ ${ }^{13]}$ have shown that MLD is an essential predictor of RP $\geq$ grade 2 . The incidence of RP has an obvious correlation with GTV dose and PTV ${ }^{[14]}$. Vogeliuset et al. ${ }^{[15]}$ reported that both GTV and PTV dose were critical factors affecting the occurrence of RP. In this study, univariate analysis showed that $V_{5}-\mathrm{V} 40, \mathrm{MLD}, \mathrm{GTV}, \mathrm{PTV}, \mathrm{GTV} /$ total lung volume, and PTV/total lung volume were associated with the occurrence of RP at all levels. Multivariate results showed that $V_{5}$ was an independent risk factor for RP $\geq$ grade 1 and $\geq$ grade 3 , and MLD was an independent risk factor for RP $\geq$ grade 2 . However, the traditional prediction parameter $V_{20}$ does not reflect the actual prediction efficiency. The reason is that $1 / 4$ of the enrolled patients adopted TOMO technology to effectively reduce the lung volume in the high-dose area; in addition, we may have strict restrictions on $\mathrm{V}_{20}$ when making the actual physical plan.

In previous studies, most patients underwent common RT, while the mainstream RT technology has been image-guided precise RT (e.g., 3DCRT, IMRT, TOMO) in recent years. The improvement of RT technology has brought about changes in lung-dose volume parameters, but the relationship between this change and RP is still uncertain. MD Anderson Tumor Center analysis ${ }^{[16]}$ showed that IMRT could significantly reduce $V_{20}$, but $V_{5}$ was increased simultaneously. Although $\mathrm{V}_{20}$ decreased, the probability of RP did not change. TOMO technology has been widely used in the RT of thoracic tumours. Some studies ${ }^{[17,18]}$ suggest that TOMO is safe in the treatment of lung cancer. EC studies ${ }^{[19,20]}$ compared lung dose parameters by selecting a few patients and designing several different physical plans; however, there is no real-world report on the occurrence of RP in EC after different irradiation techniques. We analysed the incidence of RP in 247 patients with EC undergoing RT. The incidence of RP $\geq$ grade 2 was $22.4 \%$ in IMRT and $16.9 \%$ in TOMO. More importantly, we found that the incidence of RP $\geq$ grade $1, \geq$ grade 2 , and $\geq$ grade 3 was not related to radiation technology. Therefore, precise RT does not decrease the incidence of RP, which was in line with a previous study [21,22].

As mentioned above, although lung dose parameters are associated with RP in patients with EC, the results reported in the literature are not uniform. Therefore, the occurrence of RP is not only related to lung dose volume parameters. We should comprehensively evaluate the clinical characteristics and treatment factors of patients. Studies ${ }^{[23]}$ have confirmed that RP is related to many factors, such as smoking status, chronic lung disease, and chemotherapy. Laucis ${ }^{[24]}$ deemed that smoking did not increase radiation-related lung toxicity. Moreover, a meta-analysis ${ }^{[25]}$ found that patients with a history of smoking or current smoking have a reduced risk of RP. We introduced the smoking index to study the relationship between patients with different smoking levels and RP. The results showed that there was no significant correlation between nonsmokers and the incidence of RP $\geq$ grade 1 and RP $\geq$ grade 2 in patients with different degrees of smoking. However, RP $\geq$ grade 3 was significantly increased in patients with a smoking index $>$ 400. The reason may be related to the complications of COPD caused by severe smoking. Zhang et al. ${ }^{[26]}$ found that for smokers with severe chronic lung disease, smoking has become an adverse factor of RP. The severity of smoking is an important influencing factor of severe RP. However, when the effects of tobacco are ignored, the results may be different from the perspective of COPD or essential pulmonary function. A previous report ${ }^{[27]}$ based on stereotactic 
body radiotherapy (SBRT) showed that the risk of RP in COPD and non-COPD patients was similar. Wang J et al. [28] analyzed 260 patients with stage $\otimes-\otimes$ non-small cell lung cancer who received conventional RT and found that there was no increase in the incidence of grade $\geq 2 \mathrm{RP}$ in patients with poor basic lung function due to COPD. Furthermore, in their study, patients with symptomatic RP had higher basal of forced exhalation in the first second (FEV1\%) (71.7\% vs. $65.9 \%, p=0.077$ ) than those without it. In our study, EC patients with COPD were unlikely to develop RP $\geq$ grade 2 and $\geq 3$ after RT, although this correlation was found in patients with RP $\geq$ grade 1. Grade 1 RP is an asymptomatic radiological change according to CTCAE criteria, clinical treatment is not required even when it occurs. Therefore, we should pay more attention to the relationship between COPD and symptomatic RP ( $\geq$ grade 2 ).

Chemoradiotherapy is the dominant mode of nonsurgical treatment for EC. Thus, the effect of chemotherapy on RP in patients with EC is also a concern. Whereas there are few reports in this area. In lung cancer, we can see that the research results of the effect of chemotherapy on RP are not consistent. Kishida et al. ${ }^{[29]}$ reported that RT combined with paclitaxel drugs increased the incidence of RP in lung cancer patients, but Shaikh et al. ${ }^{[30]}$ concluded that concurrent paclitaxel chemotherapy during RT did not affect RP. In our study, 205 patients (82.9\%) received chemotherapy, including induction chemotherapy, concurrent chemotherapy, and consolidation chemotherapy. However, no correlation was found between RP and chemotherapy. Considering that our study is not a large sample, this result is not enough to confirm that chemotherapy does not induce or aggravate RP after radiotherapy for EC.

As a retrospective study, this study still has some limitations. First, the sample size of the included group was relatively small, while the RP influencing factors involved were relatively large, which may cause deviation in the results. Second, the follow-up observation time was relatively short, and there was no correlation analysis for the patients who may have radiation-induced pulmonary fibrosis beyond the follow-up period. Third, it is useful to find the cut-off value of the factors that have significant influence on RP by ROC curve, but the AUCs of the three factors in this study are less than 0.8 , so the prediction efficiency is insufficient. This seems to be an insurmountable problem in current clinical work. Fortunately, in my in-depth research ${ }^{[31]}$, the RP prediction model was established by using CT radiomics analysis technology combined with clinical characteristics and dosimetric parameters, which made up for the insufficient of lower AUCs prediction in the current study. Finally, the choice of RT technology was not randomization but considered the size of the tumour target area or patient economy and other factors, which may affect the experimental results.

\section{Conclusions}

We comprehensively discussed the influencing factors of RP in patients with thoracic EC who received RT. Different lung dose-volume parameters have an essential influence on the occurrence of RP. In the implementation of EC radiation therapy, we should pay attention to dosimetry parameters such as lung $\mathrm{V}_{5}$ and MLD, regardless of which radiotherapy technique is used. In addition, we cannot ignore the significant role of the index of smoking and essential pulmonary function.

\section{Declarations}

Ethics Approval and Consent to Participate: These issues are not applicable for this review.

Consent for publication: Not applicable.

Availablility Of Data And Material : The datasets /or image are available from the corresponding author on reasonable request. 
Competing interests: The authors of this manuscript declare no relationships with any companies, whose products or services may be related to the subject matter of the article.

Funding: This study has received funding by National Key Research Program of China (grant number 2016YFC0904700); National Natural Science Foundation of China (grant number 81773287); Key Research Development Program of Shandong Province (grant number 2016GSF201093).

Authors Contributions $\square A l l$ authors made a significant contribution to the work reported, whether that is in the conception, study design, acquisition of data, analysis and interpretation; took part in drafting, revising or critically reviewing the article; gave final approval of the version to be published; have agreed on the journal to which the article has been submitted; and agree to be accountable for all aspects of the work.

Acknowledgments: This manuscript was edited by American Journal Experts (AJE) Language Editing Services.

\section{References}

1. Bray F, Ferlay J, Soerjomataram I, Siegel RL, Torre LA, et al. Global cancer statistics 2018: GLOBOCAN estimates of incidence and mortality worldwide for 36 cancers in 185 countries. CA Cancer J Clin. 2018;68:394-424.

2. Ajani JA, D'Amico TA, Bentrem DJ, Chao J, Corvera C, et al. Esophageal and Esophagogastric Junction Cancers, Version 2.2019,NCCN Clinical Practice Guidelines in Oncology. J Natl Compr Canc Netw. 2019;17(7):855-83.

3. Lin SH, Wang L, Myles B, Thall PF, Hofstetter WL,et al. Propensity score-based comparison of long-term outcomes with 3-dimensional conformal radiotherapy vs intensity-modulated radiotherapy for esophageal cancer. Int $\mathrm{J}$ Radiat Oncol Biol Phys. 2012;84(5):1078-85.

4. Senan S, Rusthoven CG, Slotman BJ, Siva S, et al. Progress in radiotherapy for regional and oligometastatic disease in 2017. J Thorac Oncol. 2018;13(4):48.

5. Wang DQ, Shi J, Liang SH, Lu SY, Qi XJ, et al. Dose-volume histogram parameters for predicting radiation pneumonitis using receiver operating characteristic curve. Clin Transl Oncol. 2013;15(5):364-9.

6. Tonison JJ, Fischer SG, Viehrig M, Welz S, Boeke S, et al. Radiation pneumonitis after intensity-modulated radiotherapy for esophageal cancer: institutional data and a systematic review. Sci Rep. 2019;9:2255.

7. Palma DA, Senan S, Tsujino K, Barriger RB, Rengan R, et al. Predicting radiation pneumonitis after chemoradiation therapy for lung cancer: an international individual patient data meta-analysis. Int J Radiat Oncol Biol Phys. 2013;85(2):444-50.

8. Simone CB. Thoracic radiation normal tissue injury. Semin Radiat Oncol. 2017;27(4):370-7.

9. Chargari C, Riet F, Mazevet M, Morel E, Lepechoux C, et al. Complications of thoracic radiotherapy. Presse Med. 2013;42:e342-51.

10. Luna JM, Chao HH, Diffenderfer ES, Valdes G, Chinniah C, et al. Predicting radiation pneumonitis in locally advanced stage II-III non-small cell lung cancer using machine learning. Radiother Oncol. 2019;133:106-12.

11. Jin X, Lin B, Chen D, Li L, Han C,et al. Safety and outcomes of volumetric modulated arc therapy in the treatment of patients with inoperable lung cancer. J Cancer. 2019;10(13):2868-73.

12. Katsui K, Ogata T, Watanabe K, Katayama N, Soh J,et al. Dose-volume parameters predict radiation pneumonitis after induction chemoradiotherapy followed by surgery for non-small cell lung cancer: a retrospective analysis. BMC Cancer. 2019;19(1):1144.

13. Pan WY, Bian C, Zou GL, Zhang CY, Hai P, et al. Combing NLR,V20 and mean lung dose to predict radiation induced lung injury in patients with lung cancer treated with intensity modulated radiation. therapy

Page $12 / 14$ 
chemotherapyOncotarget. 2017;8(46):81387-93.

14. Tanabe S, Myojin M, Shimizu S, Fujino M, Takahashi H,et al. Dose-volume analysis for respiratory toxicity in intrathoracic esophageal cancer patients treated with definitive chemoradiotherapy using extended fields. J Radiat Res. 2013;54:1085-94.

15. Vogelius IS, Westerly DC, Cannon GM, Mackie TR, Mehta MP, et al. Intensitymodulated radiotherapy might increase pneumonitis risk relative to three-dimensional conformal radiotherapy in patients receiving combined chemotherapy and radiotherapy: a modeling study of dose dumping. Int J Radiat Oncol Biol Phys. 2011;80:893-9.

16. Asakura H, Hashimoto T, Zenda S, Harada H, Hirakawa K,et al. Analysis of dose-volume histogram parameters for radiation pneumonitis after definitive concurrent chemoradiotherapy for esophageal cancer.Radiother Oncol, 2010, 95(2):240-244.

17. Arcangeli S, Agolli L, Portalone L, Migliorino MR, Lopergolo MG,et al. Patterns of CT lung injury and toxicity after stereotactic radiotherapy delivered with helical tomotherapy in early stage medically inoperable. NSCLCBr J Radiol. 2015;88(1048):20140728.

18. Kim Y, Hong SE, Kong M, Choi J,et al. Predictive factors for radiation pneumonitis in lung cancer treated with helical tomotherapy. Cancer Res Treat. 2013;45(4):295-302.

19. Nguyen NP, Krafft SP, Vinh-Hung V, Vos P, Almeida F,et al. Feasibility of tomotherapy to reduce normal lung and cardiac toxicity for distal esophageal cancer compared to three-dimensional radiotherapy. Radiother Oncol. 2011;101(3):438-42.

20. Zhang Y, Wang H, Huang X, Zhang Q, Ren R,et al. Dosimetric comparison of TomoDirect, helical tomotherapy, VMAT, and ff-IMRT for upper thoracic esophageal carcinoma. Med Dosim. 2019;44(2):167-72.

21. Haefner MF, Lang K, Verma V, Koerber SA, UhImann L,et al. Intensity-modulated versus 3-dimensional conformal radiotherapy in the definitive treatment of esophageal cancer: comparison of outcomes and acute toxicity. Radiat Oncol. 2017;12(1):131.

22. Shridhar R, Almhanna K, Meredith KL, Biagioli MC, Chuong MD, et al. Radiation therapy and esophageal cancer. Cancer Control. 2013;20:97-110.

23. Giuliani ME, Lindsay PE, Kwan JY, Sun A, Bezjak A,et al. Correlation of dosimetric and clinical factors with the development of esophagitis and radiation pneumonitis in patients with limited stage small-cell lung carcinoma. Clin Lung Cancer. 2015;16(3):216-20.

24. Laucis AM, Sun Y, Schipper M, MaurinoC,Jolly S. Active smoking is not associated with increased radiationinduced toxicity in locally advanced lung cancer patients. Int J Radiat Oncol Biol Phys. 2018;102(3):231-1.

25. Vogelius IR, Bentzen SM. A literature-based meta-analysis of clinical risk factors for development of radiation induced pneumonitis. Acta Oncol. 2012;51(8):975-83.

26. Zhang XJ, Sun JG, Sun J, Wang XX, Wu L,et al. Prediction of radiation pneumonitis in lung cancer patients: a systematic review. J Cancer Res Clin Oncol. 2012;138(12):2103-16.

27. Takeda A, Kunieda E, Ohashi T, Aoki Y, Oku Y, et al. Severe COPD is correlated with mild radiation pneumonitis following stereotactic body radiotherapy. Chest. 2012;141(4):858-66.

28. Wang J, Cao J, Yuan S, et al. Poor baseline pulmonary function may not increase the risk of radiation induced lung toxicity. Int J Radiat Oncol Biol Phy. 2013;85(3):798-804.

29. Kishida Y, Tsushima T, Endo M, Hamauchi S, Todaka A, et al. Risk analysis of pneumonitis in taxane therapy after chemoradiotherapy for patients with metastatic or recurrent esophageal cancer. Am J Clin Oncol. 2018;41(1):415 . 
30. Shaikh T, Churilla TM, Monpara P, Scott WJ, Cohen SJ,et al. Risk of radiation pneumonitis in patients receiving taxane-based trimodality therapy for locally advanced esophageal cancer. Pract Radiat Oncol. 2016;6(6):388-94.

31. Du F, Tang N, Cui Y, Wang W, Zhang Y, et al. A novel nomogram model based on cone beam CT radiomics analysis technology for predicting radiation pneumonitis in esophageal cancer patients undergoing radiotherapy. Front Oncol,2020,Dec 17;10:596013.

\section{Figures}
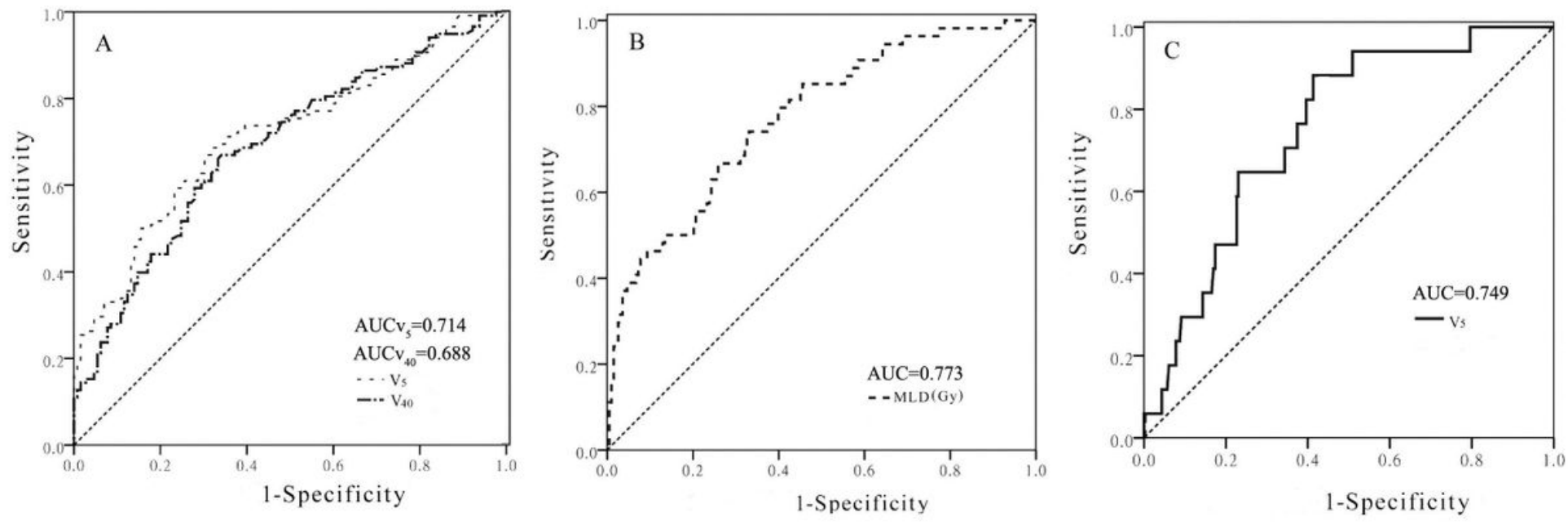

Figure 1

ROC curve for predicting the corresponding grade RP (Figure 1). A) RP $\geq$ grade 1 (Figure. B) RP $\geq$ grade 2 (Figure. C) $\mathrm{RP} \geq$ grade 3 